\title{
Strain development in a filled epoxy resin curing under constrained and unconstrained conditions as assessed by Fibre Bragg Grating sensors
}

\author{
M. Harsch ${ }^{* 1}$, J. Karger-Kocsis ${ }^{2}$, F. Herzog ${ }^{1}$ \\ ${ }^{1}$ Robert Bosch GmbH, Robert Bosch Straße, 87542 Blaichach, Germany \\ 2Institut für Verbundwerkstoffe GmbH, Erwin-Schrödinger-Straße, 67663 Kaiserslautern, Germany
}

Received 22 February 2007; accepted in revised form 5 April 2007

\begin{abstract}
The influence of adhesion to the mould wall on the released strain of a highly filled anhydride cured epoxy resin (EP), which was hardened in an aluminium mould under constrained and unconstrained condition, was investigated. The shrinkage-induced strain was measured by fibre optical sensing technique. Fibre Bragg Grating (FBG) sensors were embedded into the curing EP placed in a cylindrical mould cavity. The cure-induced strain signals were detected in both, vertical and horizontal directions, during isothermal curing at $75^{\circ} \mathrm{C}$ for 1000 minutes. A huge difference in the strain signal of both directions could be detected for the different adhesion conditions. Under non-adhering condition the horizontal and vertical strain-time traces were practically identical resulting in a compressive strain at the end of about $3200 \mathrm{ppm}$, which is a proof of free or isotropic shrinking. However, under constrained condition the horizontal shrinkage in the EP was prevented due to its adhesion to the mould wall. So, the curing material shrunk preferably in vertical direction. This resulted in much higher released compressive strain signals in vertical (10 $430 \mathrm{ppm})$ than in horizontal (2230 ppm) direction. The constrained cured EP resins are under inner stresses. Qualitative information on the residual stress state in the molding was deduced by exploiting the birefringence of the EP.
\end{abstract}

Keywords: thermosetting resins, cure-induced strain, Fibre Bragg Grating sensor, adhesion, shrinkage

\section{Introduction}

Engineering parts produced of thermosets are widely used in different fields like the automotive, electronic, aircraft and sports industry. Their cure as neat materials or matrices of various composites often takes place in a mould to the wall of which the curing material strongly adheres. Furthermore, thermosets may get under constraints when they are employed together with other materials (metals, ceramics) having different thermal expansion behaviour than the curing resin. During curing the adhesion to the surrounding material plays a key role in terms of induced strains and, thus, stresses. A non-adhering thermoset cures under free-shrink-

${ }^{*}$ Corresponding author, e-mail: margitharsch@yahoo.de

(c) BME-PT and GTE ing condition, whereas adhesion to the mould wall prohibits unhindered contraction of resin due to which inner stresses emerge in the curing material. In an early curing stage the thermoset is 'soft' with a high potential of 'elastic' deformation and relaxation. With increasing crosslinking density the flexibility of the material decreases. Under adhering condition this leads to the build-up of inner stresses which may cause low performance, shape distortion, warpage or even failure due to matrix cracking and delamination. The technique of fibre optical measurements provides the opportunity to gain information about the cure-induced strain and, thus, to enquire the stress resistance of a material. The Fibre Bragg Grating (FBG) sensing technique 
offers numerous advantages over the traditionally used methods. Due to its small diameter (ca. $125 \mu \mathrm{m})$ and its chemical inertness the effective network building within the EP is hardly affected. Another advantage of this FBG method is that the outcome is independent of the geometry of the sample and of the thermal profile set for its curing. Many authors applied this method for the 'health monitoring' of composite structures [1-7]. Further studies aim at the use of fibre optical technique to characterize cure of resins [8-19]. In our earlier works $[8,9]$ we reported about the use of FBG sensors to monitor the cure-induced strain development in epoxy resins (EP). Beside the detection of characteristic phenomena like gelation and vitrification, the coefficient of thermal expansion was determined and parameters of the cure regime on the released strain were investigated. As reported there, FBG sensors make use of the Bragg reflectivity to detect axial changes along the optical fibre [20]. The FBG is a segment of the optical fibre with a periodic modulation of the core refractive index. Its principle is shown schematically in Figure 1.

A broadband spectrum light (Incident Spectrum) is guided along the core of the optical fibre. One particular wavelength $\lambda_{B}$ is reflected at the gratings within the fibre core whereas the other part of the incident spectrum is transmitted through the fibre. The wavelength reflected by the FBG, the Bragg wavelength $\lambda_{B}$, is dependent on the effective refractive index $n_{\text {eff }}$ of the fibre and the grating periodic spacing $\Lambda$, according to the Bragg condition (1):

$\lambda_{B}=2 \cdot n_{e f f} \Lambda$

Strain or temperature modulations cause changes in the effective refractive index or the periodic spac-

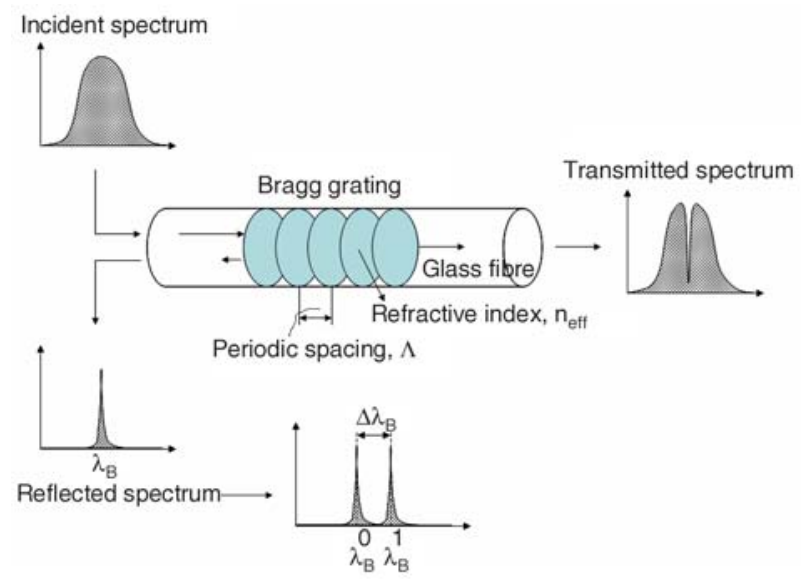

Figure 1. Principle of Fibre Bragg Grating sensor ing and thereby entail a shift in the Bragg grating wavelength $\Delta \lambda_{B}$ (c.f. Figure 1). This shift can be detected by an optical spectrum analyzer with an accuracy of $1 \mathrm{pm}$. Further information about the theoretical background of the FBG theory as well as the measuring setup of the Bragg wavelength shift, is given in the literature $[8-9,20]$.

During thermoset cure the material solidifies and contracts due to volume shrinkage. The development of compressive stresses can be measured by a shift in the reflected Bragg wavelength. Simultaneous measurements of the actual temperature allow us to eliminate the contribution of thermal changes on the wavelength signal. Adhesion to the wall may hinder the free shrinkage during cure. Therefore, the 'surroundings' influence the cure-induced strain signal to a great extent. Giordano et al. [17] reported about the strain signal measured by FBG after certain production steps of an EP resin. Within the mould the material had lower compressive strain than after demoulding when further relaxation amplified the compressive stresses in the cured material.

In this study we investigated the influence of adhesion on the strain development in a curing EP resin by using the fibre optical technique. Along the centre axis of a cylindrical sample holder and perpendicular to it FBG sensors were embedded in the curing material. During isothermal cure the cureinduced released strain under adhering (constrained) and non-adhering conditions (unconstrained) were measured and compared. First strain appearance measured by the FBG was compared to the validated gel point determination, based on the rheological measurement. The residual state of stress in the EP cured under constrained and unconstrained conditions was qualitatively assessed by polarized light microscopy.

\section{Experimental part}

\subsection{Material}

The investigated material was an EP system on base of Bisphenol A (Araldite MY740, molecular weight $340.41 \mathrm{~g} / \mathrm{mol}$ ), which was cured with a mixture of two anhydrides, viz. methyl hexahydrophthalic anhydride (molecular weight $162.14 \mathrm{~g} / \mathrm{mol}$ ) and hexahydrophthalic anhydride $(154.17 \mathrm{~g} / \mathrm{mol})$. The mixing ratio of the EP resin to hardener was 100:83. Furthermore, the EP was loaded with a 
high content of inorganic filler (ca. 50 wt.\%) on base of calcium silicate and calcium carbonate. All basic materials were supplied by Huntsman, Basel, Switzerland and used as provided.

\subsection{Preparation and curing parameter}

After stirring of resin, hardener and filler the homogenous mixture was degassed and preheated to $60^{\circ} \mathrm{C}$ before filling it in a cylindrical aluminium mould (sample holder). The mould itself was preheated to $75^{\circ} \mathrm{C}$ and equipped with an FBG sensor. To make the mould non-adherent for tests under unconstrained conditions it was covered with a thin layer of silicon oil. For constrained tests, the mould surface was roughened in order to enhance the surface to which the EP could adhere. The mould was $25 \mathrm{~mm}$ in diameter and ca. $25 \mathrm{~mm}$ in height and contained about $20 \mathrm{ml}$ of the curing EP resin system. The EP was cured under isothermal condition of $75^{\circ} \mathrm{C}$ for $1000 \mathrm{~min}$.

\subsection{Fibre Bragg Grating sensing principle}

For vertical strain measurements the FBG sensor was placed along the centre axis of the cylinder in the middle of the mould cavity. Horizontal measurements were realized by placing an FBG sensor at half of the height of the cylinder perpendicular to the longitudinal axis. The setup for both FBG assemblies can be seen in Figure 2.

A broad spectrum incident light with a central wavelength of $1549 \mathrm{~nm}$ was guided along the glass fibre. The back-reflected wavelength from the Bragg Gratings was detected by an optical spectrum analyzer and transmitted to an evaluating processor unit. To consider the influence of temper-

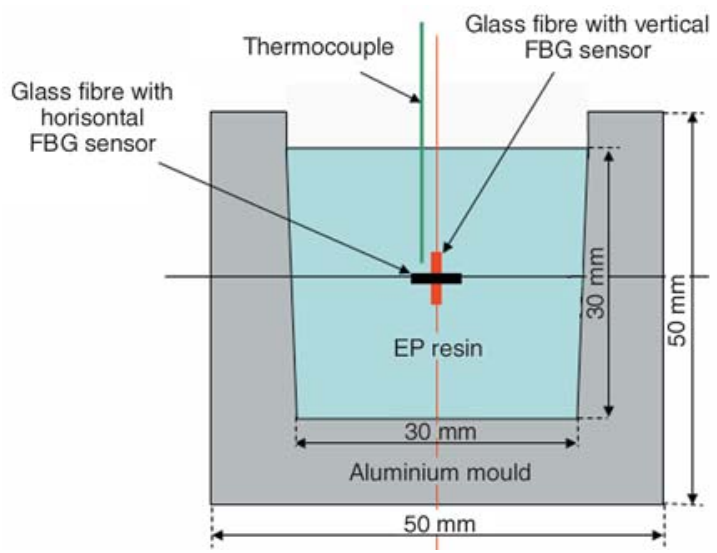

Figure 2. Setup of EP in an aluminium mould with embedded FBG sensors (vertical and horizontal) ature shifts on the strain signal, the actual temperature was measured by a thermocouple in the sample next to the FBG sensor. This contribution to the detected strain signal was eliminated in all the investigated strain signals.

\subsection{Rheological measurement}

To determine the gel point of the EP, a rheological measurement was performed on a Haake RS 1 (Thermo Electron, Karlsruhe) rheometer equipped with a thermocontroller TCP/P using $60 \mathrm{~mm}$ diameter parallel plates with a gap of $0.5 \mathrm{~mm}$. The experiment was carried out under oscillatory conditions applying an angular frequency of $10 \mathrm{rad} \mathrm{s}^{-1}$ and an amplitude of 5\%. The EP to be cured was placed into the preheated device and cured isothermally at $75^{\circ} \mathrm{C}$ for 190 minutes. The courses of the storage $\left(G^{\prime}\right)$ and loss $\left(G^{\prime \prime}\right)$ moduli were recorded as a function of the reaction time. The gel time was determined at the crossover of $G^{\prime}$ and $G^{\prime \prime}$.

\section{Results}

Strain signals in vertical (along the cylinder axis) and horizontal direction of the mould are investigated under constrained and unconstrained condition for isothermal cure of $1000 \mathrm{~min}$ at $75^{\circ} \mathrm{C}$. Whereas under non-adhering condition the material undergoes a free-shrinking process, adhesion to the mould wall prevents the unhindered contraction of the EP. This is due to chemical bonding and/or mechanical 'anchoring' of the EP to the mould wall. The latter should result in inner stresses, and in worst case, even in material cracking. The stress field can be made viewable under polarized light. Polarized light microscopic pictures taken for adhering and non-adhering conditions are shown in Figure 3.

Under constrained condition (Figure 3a) the isochromates indicate the imposed stress levels quali-

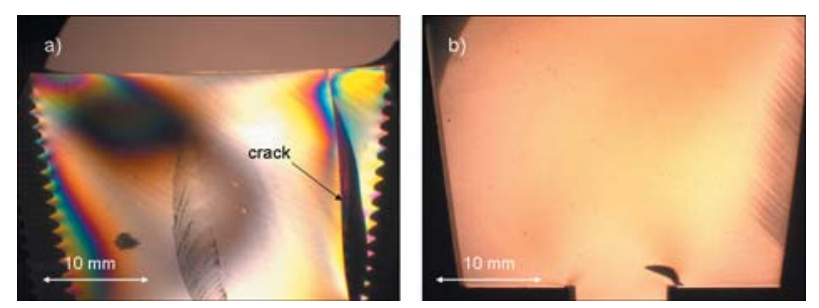

Figure 3. Sections of the EP moldings viewed under polarized light after curing with (a) and without (b) adhesion to the mould wall 


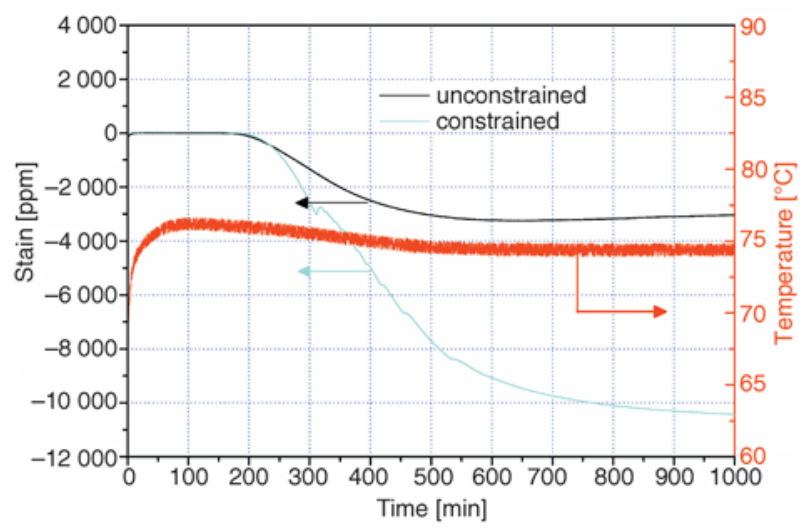

Figure 4. Strain development in the EP in vertical direction under constrained and unconstrained condition. Note that this figure contains also the related temperature-time trace

tatively - cf. colored regions. Note that on the right hand side of the moulding (arrow shows) the prevailing stresses produced a large crack. On the other hand free-shrinking condition resulted in a cured stress-free material, which can be seen in the non-coloured section of the EP molding in Figure 3b.

Strain development (strain-time curves) for the curing EP under constrained and unconstrained condition along the cylinder axis (vertical) was measured and shown together with the actual temperature in Figure 4.

As already discussed in our earlier works [8,9], at the beginning no strain signal could be detected because the curing EP was in liquid form and the cure shrinkage was relieved by liquid flow. After about 170 minutes the FBG sensor detects a strain signal for the first time. This point was assigned to the gel point of the EP where the incipient forma-

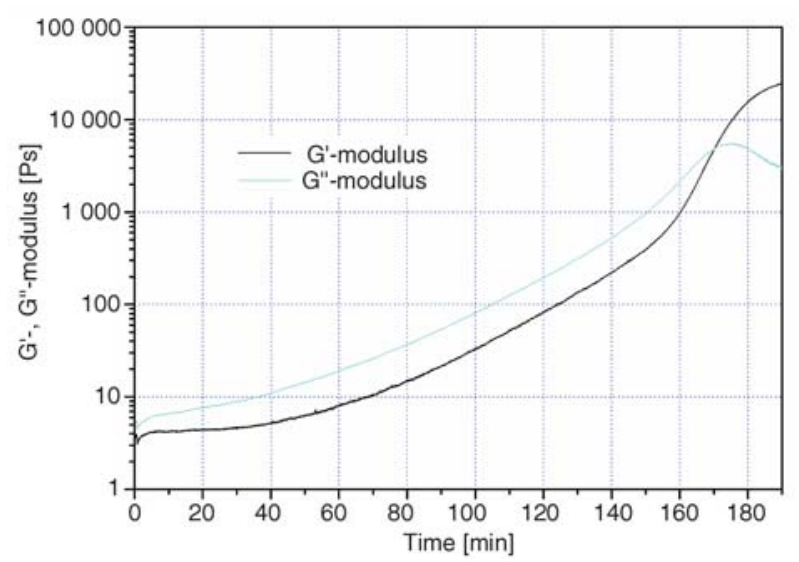

Figure 5. Reological measurement of storage $\left(G^{\prime}\right)$ and loss $\left(G^{\prime \prime}\right)$ shear moduli of the EP as a function of time under isothermal condition of $75^{\circ} \mathrm{C}$ tion of a material with infinite molecular weight occurs. The gel time of the $\mathrm{EP}$ resin at $75^{\circ} \mathrm{C}$ was also deduced from the rheological measurement. The results are shown in Figure 5 where $G^{\prime}$ and $G^{\prime \prime}$ are plotted against time.

Gelling is defined when the $\mathrm{G}^{\prime}$ - and $\mathrm{G}^{\prime \prime}$ - shear moduli are equal. As can be seen in Figure 4 this occurs after about 170 minutes. The rheology result is in very good accordance with the first strain detection by the FBG sensing technique. After gelation an almost linear build-up of compressive strain took place before tending to flatten (cf. Figure 4). This is due to the advanced crosslinking of the EP. After about 380 minutes the first deviation of the linear decreasing strain line was found to represent the vitrification of the curing EP. This was proven by differential scanning calorimetry measurements, as disclosed in our earlier reports $[8,9]$. Vitrification indicates the transformation from a rubbery to a glassy material and it occurs when the glass transition temperature $T_{g}$ of the curing material equals the isothermal curing temperature.

Under the non-adhering condition, at the end of the isothermal curing, a released strain of about $3040 \mathrm{ppm}$ was detected. In case of adhesion to the mould wall the strain signal indicated $10430 \mathrm{ppm}$ of compressive strain (cf. Figure 4). Because the adhesion to the wall is known to hinder the freeshrinkage of the EP, it is reasonable that the released strain is higher under constrained than under free-shrinking condition. Recall that for the reported measurements the FBG sensor was placed vertically in the EP resin. During crosslinking the EP contracts but radial shrinkage (i.e. horizontal) movements of the curing EP are prevented because the resin is 'fixed' to the wall. So the material flux occurs only in vertical direction where the surface is open (cf. Figure 2) and, thus, material 'movement' is possible. Correspondingly, higher compressive strain should be detectable by the FBG sensor in vertical than in horizontal direction. We suppose that the detected vertical strain under adhering condition is about three times higher than under free-shrinking condition. This is because the volume shrinkage is substituted by linear shrinkage which should contain the contribution of shrinkage of the two plane directions, as well as, the vertical contribution. In order to affirm the above speculation on the strain differences in horizontal and vertical directions the strain signal of the curing EP under adhering condition was measured simultane- 


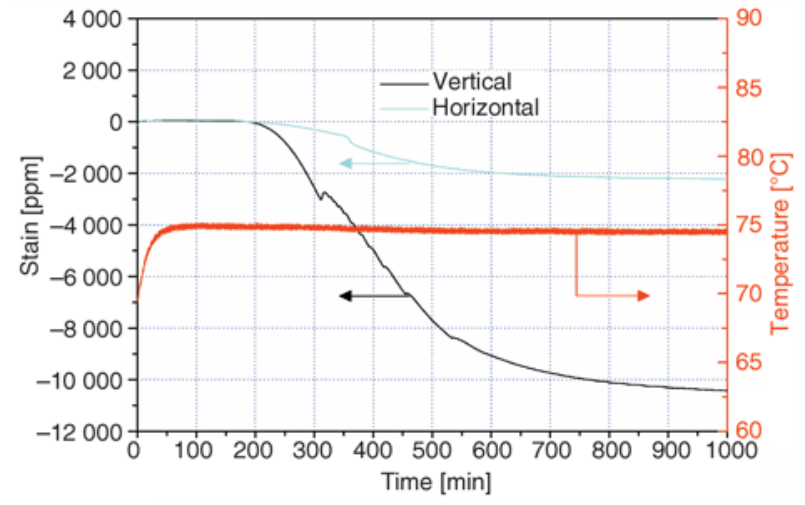

Figure 6. Strain development in the EP in vertical and horizontal directions under constrained condition. Note that this figure contains also the related temperature-time trace

ously in both, longitudinal and vertical directions (cf. Figure 6).

As can be seen in Figure 6, released strain after 1000 minutes is much lower in horizontal direction, viz. about $2230 \mathrm{ppm}$, compared to the vertical direction, which indicates $10430 \mathrm{ppm}$. The adhesion to the mould wall prevents the build-up of strong compression stresses on the horizontal FBG sensor. Nevertheless, the small FBG signal indicates that even in this direction moderate compressive stress works due to the chemical reaction-induced shrinkage. This, in fact, induces stresses in the curing material as shown qualitatively in Figure 3. A final confirmation on the reliability of strain detection under constrained and unconstrained condition can be received by the investigation of the vertical and horizontal strain development under free-shrinking condition. The corresponding strain signals, as well as the meas-

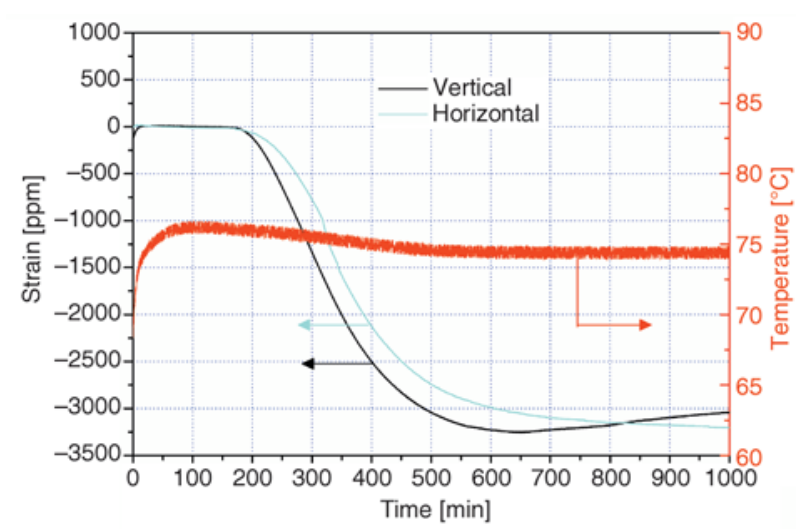

Figure 7. Strain development of the EP in vertical and horizontal directions under unconstrained condition. Note that this figure contains also the related temperature -time trace.
Table 1. Released strain in the EP after isothermal cure at $75^{\circ} \mathrm{C}$ for 1000 minutes

\begin{tabular}{|l|c|c|c|c|}
\hline Adhesion condition & \multicolumn{2}{|c|}{ Constrained } & \multicolumn{2}{c|}{ Unconstrained } \\
\hline Direction & vertical & horizontal & vertical & horizontal \\
\hline Released strain [ppm] & 10430 & 2230 & 3040 & 3200 \\
\hline
\end{tabular}

ured temperature as a function of time are shown in Figure 7.

Apparently, the investigated strain signals in vertical and horizontal directions show almost the same results with a released strain of about $3000 \mathrm{ppm}$ at the end of the experiment. The curing EP shrunk isotropically and, thus, there was practically no difference in the strain signals between vertical and horizontal FBG measurements. Whereas the vertical direction is favoured for material's movement for cure under constrained condition (c.f. Figure 6) in the present set-up, non-adhering cure conditions lead to an isotropic compression (c.f. Figure 7) due to cure-shrinkage.

The entire results of the released strain in horizontal and vertical direction after curing the EP for 1000 minutes isothermally at $75^{\circ} \mathrm{C}$ under constrained and unconstrained condition are summarized in Table 1.

\section{Conclusions}

The cure-induced strain development in a highly filled, anhydride hardened epoxy (EP) system was investigated by considering the adhesion to the moulding wall. FBG sensors were embedded vertically and horizontally in a cylindrical aluminium mould, which was made adhering (constrained) or non-adhering (unconstrained) to the EP resin. Unconstrained condition resulted in a free shrinkage of the curing EP in all directions, whereas adhesion to the mould wall prevented the unhindered contraction of the material during crosslinking. In the latter case the EP could flow only in vertical direction owing to the open surface of the mould in our setup. The strain values were the highest under constrained condition in vertical direction (10 $430 \mathrm{ppm})$ which was the preferred flow direction. Note that the lateral (horizontal) movement of the curing material was restricted under constrained conditions leading to a small value of released strain $(2230 \mathrm{ppm})$. By contrast, curing under free-shrinking condition (i.e. in case of a non-adhering mould wall) resulted in almost 
equal horizontal (3200 ppm) and vertical (3040 ppm) strain signals since isotropic shrinking was afforded. In all cases first strain appearance measured by the FBG was found to agree very well with the known determination of gel point by rheological measurement. The residual stresses in the cured moldings were made visible by taking polarized light microscopic images from sections of the samples.

\section{References}

[1] Wang H., Ogin S. L., Thorne A. M., Reed G. T.: Interaction between optical fibre sensors and matrix cracks in cross-ply GFRP laminates. Part 2: Crack detection. Composites Science and Technology, 66, 2367-2378 (2006).

[2] Ling H. Y., Lau K. T., Cheng L., Su Z.: Mode II fracture behaviour monitoring for composite laminates using embedded fibre Bragg grating sensor. Composite Structures, 76, 88-93 (2006).

[3] Lau K. T., Yuan L., Zhou L. M., Xu J., Woo C. H.: Strain monitoring in FRP laminates and concrete beams using FBG sensors. Composite Structures, 51, 9-20 (2001).

[4] Murukeshan V. M., Chan P. Y., Ong L. S., Seah L. K.: Cure monitoring of smart composites using fiber Bragg grating based embedded sensors. Sensors and Actuators, 79, 153-161 (2000).

[5] Botsis J., Humbert L., Colpo F., Giaccari P.: Embedded fiber Bragg grating sensor for internal strain measurements in polymeric materials. Optics and Lasers in Engineering, 43, 491-510 (2005).

[6] Yashiro S., Okabe T., Takeda N.: Damage identification in a holed CFRP laminate using a chirped fiber Bragg grating sensor, Composites Science and Technology, 67, 286-295 (2007).

[7] Okabe Y., Yashiro S., Tsuji R., Mizutani T., Takeda N.: Effect of thermal residual stress on the reflection spectrum from fiber Bragg grating sensors embedded in CFPR laminates. Composites: Part A, 33, 991-999 (2002).

[8] Harsch M., Karger-Kocsis J., Herzog F.: Influence of cure regime on the strain development in an epoxy resin as monitored by fibre Bragg grating (FBG) sensor. Macromolecular Materials and Engineering, in press (2007).

[9] Harsch M., Karger-Kocsis J. Herzog F.: Monitoring of cure-induced strain of an epoxy resin by fibre Bragg grating sensor. Journal of Applied Polymer Science, submitted (2007).
[10] Colpo F., Humbert L., Giaccari P., Botsis J.: Characterization of residual strains in an epoxy block using an embedded FBG sensor and the OLCR technique. Composites: Part A, 37, 652-661 (2006).

[11] Leng J. S., Asundi A.: Real-time cure monitoring of smart composite materials using extrinsic Fabry-Perot interferometer and fiber Bragg grating sensors. Smart Material Structures, 11, 249-255 (2002).

[12] Quintero S. M. M., Quirino W. G., Triques A. L. C., Valente L. C. G., Braga A. M. B., Achete C. A., Cremona M.: Thin film stress measurements by fiber optic strain gage. Thin Solid Films, 494, 141-145 (2006).

[13] O’Dwyer M. J., Maistros G. M., James S. W., Tatam R. P., Partridge I. K.: Relating the state of cure to the real-time internal strain development in a curing composite using in-fibre Bragg gratings and dielectric sensors. Measurement Science and Technology, 9, 11531158 (1998).

[14] Chehura E., Skordos A. A., Ye C-C., James S. W., Partridge I. K., Tatam R. P.: Strain development in curing epoxy resin and glass fibre/epoxy composites monitored by fibre Bragg grating sensors in birefringent optical fibre. Smart Material Structures, 14, 354-362 (2005)

[15] Dawood T. A., Shenoi R. A., Sahin M.: A procedure to embed fibre Bragg grating strain sensors into GFRP sandwich structures. Composites: Part A, 38, 217-226 (2007).

[16] Cusano A., Breglio G., Giordano M., Calabro A., Cutolo A., Nicolais L.: Optoelectronic characterization of the curing process of thermoset-based composites. Journal of Optics A: Pure and Applied Optics, 3, 126-130 (2001).

[17] Giordano M., Laudati A., Nasser J., Nicolais L., Cusano A., Cutolo A.: Monitoring by a single fiber Bragg grating of the process induced chemo-physical transformations of a model thermoset. Sensors and Actuators A, 113, 166-173 (2004).

[18] Antonucci V., Cusano A., Giordano M., Nasser J., Nicolais L.: Cure-induced residual strain build-up in a thermoset resin. Composites: Part A, 37, 592-601 (2006).

[19] Antonucci V., Giordano M., Cusano A., Nasser J., Nicolais L.: Real time monitoring of cure and gelification of a thermoset matrix. Composites Science Technology, 66, 3273-3280 (2006).

[20] Othonos A., Kalli K.: Fiber Bragg Gratings. Artech House, London (1999). 